\title{
High Resolution Imaging for Bistatic P and VHF Band SAR
}

\author{
A V Borisenkov ${ }^{1}$ and O V Goriachkin ${ }^{1}$ \\ ${ }^{1}$ Povolzhskiy State University of Telecommunications and Informatics, Lev Tolstoy str. 23, \\ Samara, Russia, 443010
}

\begin{abstract}
In the article bistatic SAR imaging algorithm for P and VHF bands is described. The main feature of the system is that it uses two probing signals emitted simultaneously in two different frequency bands. As a consequence the spatial resolution of the proposed SAR can be potentially increased up to two meters. The results of full-scale ground experiment involving the proposed SAR are presented in the article.
\end{abstract}

\section{Introduction}

Spaceborne Synthetic Aperture Radars (SAR) for Earth remote sensing using P and VHF bands have been widely discussed over the last several years. Radar images for these bands contain the information about the reflection coefficient inside vegetation and soil [1,2]. Wideband SAR remote sensing using 20-500 MHz band and 100+ MHz bandwidth onboard unmanned aerial vehicles (UAV) and other mobile carriers opens up new application possibilities for Earth remote sensing and defence systems. The most interesting of these possibilities is the detection of small objects concealed by foliage or located below ground.

Airborne ground penetrating radars are known since the late 80's of the 20th century. In the year 1988 JPL started the AIRSAR platform development for VHF band imaging. Around the same time Stanford Research Institute and Swedish Defence Research Agency begun the development of longwave radars. In Soviet Union similar projects were started in the year 1989 by the Institute of Radio-engineering and Electronics in Kharkiv and by the Central Construction Bureau in Samara under the leadership of Professor A.I. Kalmykov.

Currently there exist several airborne radar systems that allow detection through foliage and below ground: Carabas FOPEN Demonstrator developed by Saab Group, Sweden; FOPEN Reconnaissance, Surveillance, Tracking and Engagement Radar; TRACER - a dual band (UHF/VHF) radar; Boeing A160T - a P-band radar operating onboard an unmanned helicopter [2-5].

From the year 2005 to the present time a scientific group of the Earth Radar Remote Sensing Center (ERRSC PSUTI, Samara) have been developing novel remote sensing systems. From global expensive space and aviation systems to local and relatively cheap but sufficient quality technologies for UAVs, ground transports or stationary ground-based infrastructures [4-6].

In contrast to works [1-3] in this paper an imaging algorithm for Bistatic SAR (BiSAR) using P and VHF bands simultaneously is described. The SAR was developed by ERRSC PSUTI during the years 2013-2017. Field test results for the SAR are presented. The test was aimed at obtaining ultra-high resolution images utilizing the combined $80 \mathrm{MHz}$ data composed from $30 \mathrm{MHz} \mathrm{P}$ band and $50 \mathrm{MHz}$ VHF band data. 


\section{Equipment of BiSAR}

BiSAR radio equipment consists of two pulse transmitters which can produce stable chirp and/or PSK signals at carrier frequencies of 435 and $145 \mathrm{MHz}$. Signal bandwidth can be adjusted from 1 to 30 $\mathrm{MHz}$ in $\mathrm{P}$ band and from 25 to $50 \mathrm{MHz}$ in VHF band. The equipment is mounted on a car and utilizes autonomous primary and secondary power sources.

Both onboard transmitter and ground-based receiver use a Yagi-Uda antenna. Ground-based stationary receiver consists of two dual channel tuned radio frequency receivers with up to $110 \mathrm{~dB}$ power gain. Each receiver records in-phase and quadrature components of a signal at $200 \mathrm{MHz}$ frequency using digital representation for subsequent digital signal processing. Receiving antenna height can vary from 8 to 12 meters.

The described configuration of the radar system allows to acquire synchronous radio images for $\mathrm{P}$ and VHF bands and also a combined high resolution image using 30 and $50 \mathrm{MHz}$ signals simultaneously. The idea of the method is illustrated by Fig. 1.

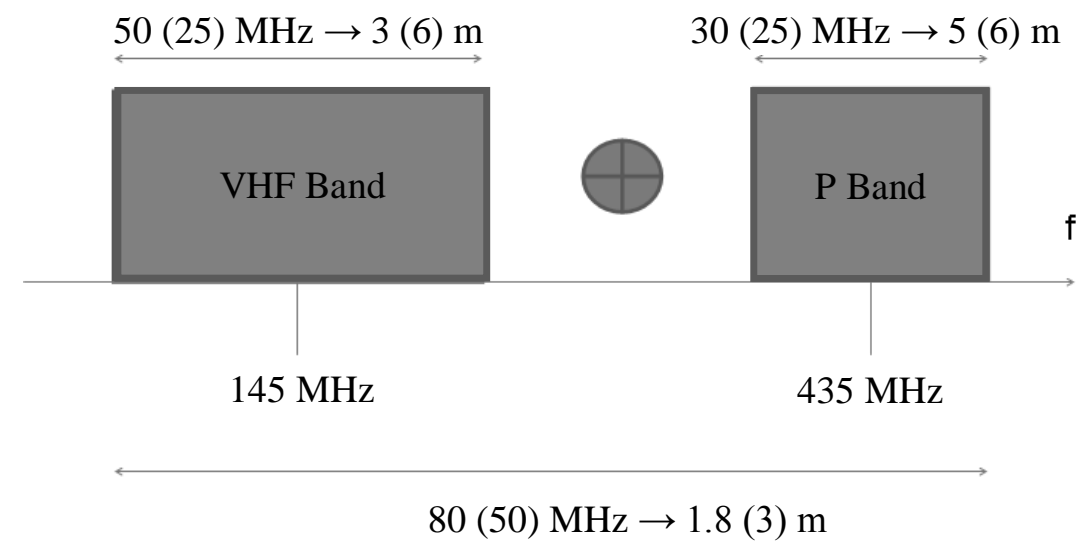

Figure 1. Combination of two frequency bands for SAR range resolution increase.

\section{Imaging algorithm}

In the paper the specifics of radar imaging algorithm for ground-based experiment are considered. For the BiSAR modification in question Signal-to-Noise Ratio (SNR) for direct channel is high enough, so we can use a simple threshold method to detect probing signals in the presence of noise and reflected signals, which provides the required synchronization of each of the frequency bands independently.

We can describe the steps of radar imaging for each of the frequency bands as follows:

1. Synchronous detection and band-pass filtering of both direct and reflected BiSAR signals recorded by Analog-to-Digital Converter (ADC);

2. Range compression using digital matched filter with chirp or PSK pulses of both direct and reflected BiSAR channels for each of the frequency bands;

3. Synchronization of the direct and reflected channels by producing a file of compressed probing signal sample indices;

4. Producing of two-dimensional (2D) radio hologram files using direct and reflected signals in $P$ and VHF band;

5. Making of a combined 2D radio hologram file for frequency shift of $f_{S}$ (8-25 $\mathrm{MHz}$ during the experiment);

6. Computing of a combined dual frequency radio image using the following formula:

$$
\begin{gathered}
\hat{\dot{\xi}}(x, y)=\int_{t_{1}}^{t_{2}} G_{\Sigma}(t, x, y) s_{2}^{*}\left(t-\tau_{2}(t, x, y)+\tau_{1}(t)\right) \dot{s}_{2}(t) . \\
\left(\begin{array}{l}
\exp \left(-j 2 \pi\left(f_{P}-f_{s}\right)\left(\tau_{2}(t, x, y)-\tau_{1}(t)\right)\right)+ \\
+\exp \left(-j 2 \pi\left(f_{\text {VHF }}+f_{s}\right)\left(\tau_{2}(t, x, y)-\tau_{1}(t)\right)\right)
\end{array}\right) d t
\end{gathered}
$$

Here $\hat{\xi}(x, y)$ is the backscattering coefficient (radar image); $\dot{s}_{2}(t)$ is the combined 2D radio hologram signal which consists of a sequence of reflected and probing pulses after preliminary matched filtering 
at zero carrier frequency, frequency shifting and summation of the signals from the two frequency bands; $\tau_{2}(t, x, y)$ is the time delay of the signal, reflected by a point target at the coordinates $(x, y)$; $\tau_{1}(t)$ is the time offset caused by transmitter instability and estimated by synchronization system; $G_{\Sigma}(t, x, y)$ is the weight function, which depends on movement patterns of transmitting and receiving BiS AR antennas.

Fig. 2 shows a block diagram of the described imaging algorithm.

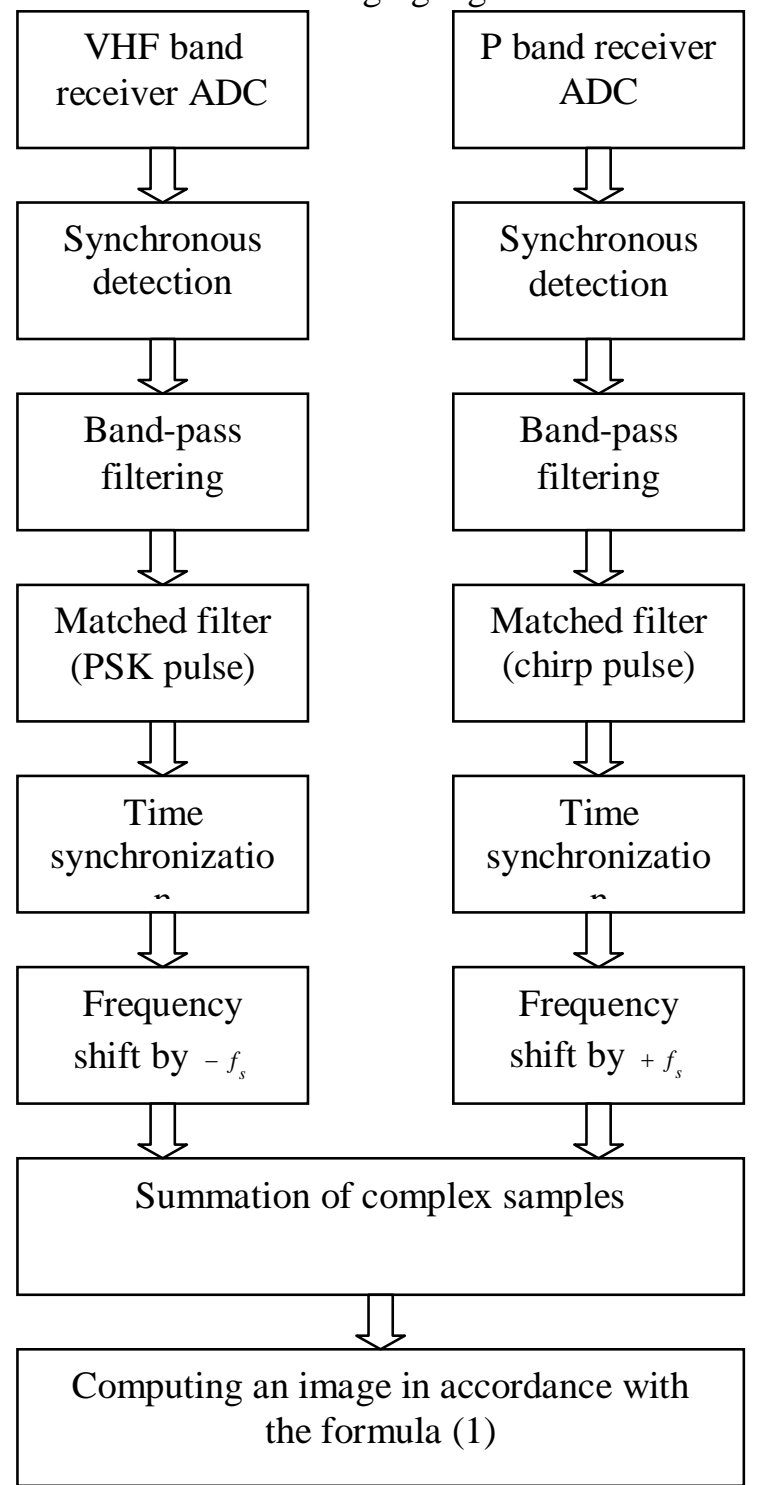

Figure 2. Block diagram of the imaging algorithm.

\section{Ground-based experiment results}

In the paper the results of the ground-based experiment involving the proposed dual frequency bistatic SAR are presented. For BiSAR system to function properly, the transmitter should be moving while emitting a signal. During the experiment we used a car moving across a bridge. The bridge height was about 20 meters. Fig. 3 shows the geometry of the experiment.

Fig. 6 shows the resulting radar image of the scene of the experiment $(4 \times 8 \mathrm{~km})$ combined with optical image of the same scene acquired via Google maps service. 


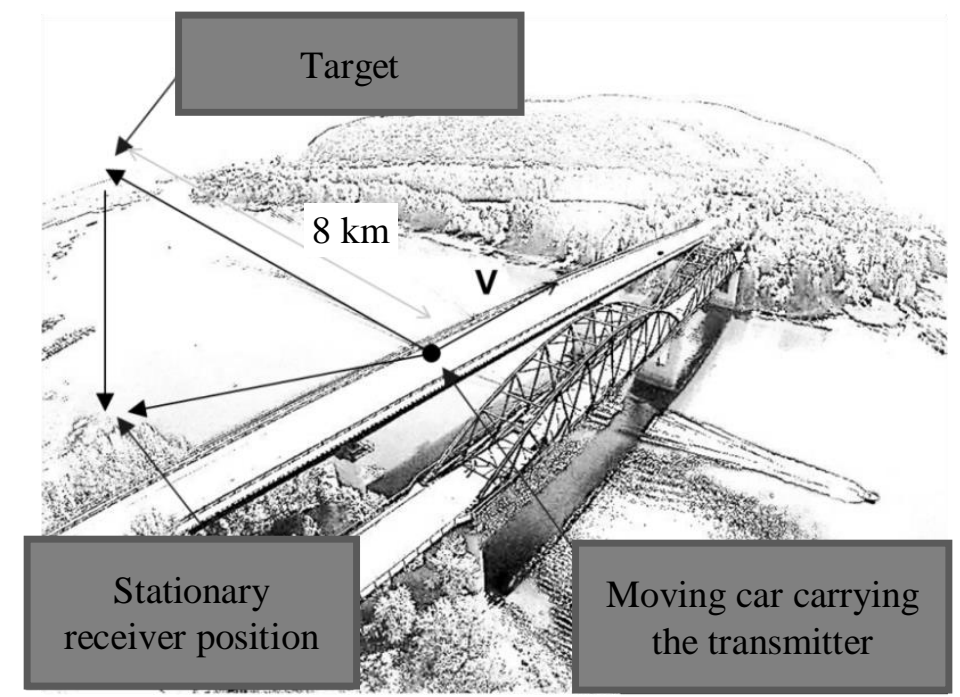

Figure 3. Ground-based experiment geometry.

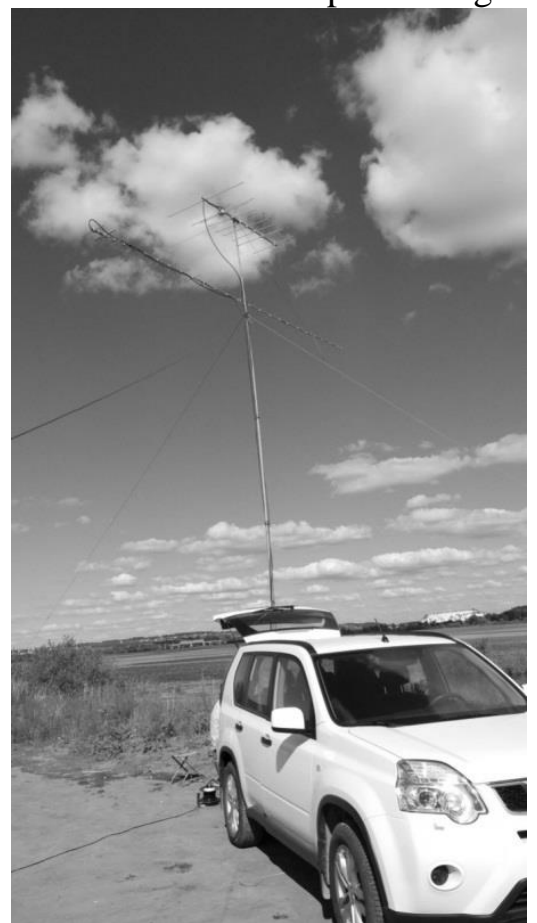

Figure 4. Stationary receiver position deployed in the field.

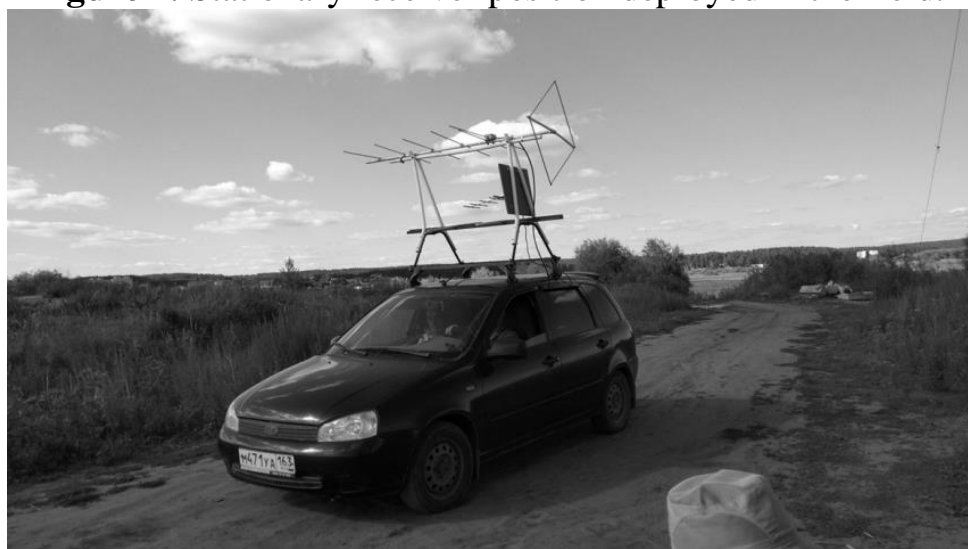

Figure 5. Moving car, carrying the transmitter across Sok River (see Fig. 6 for the scene of the experiment). 


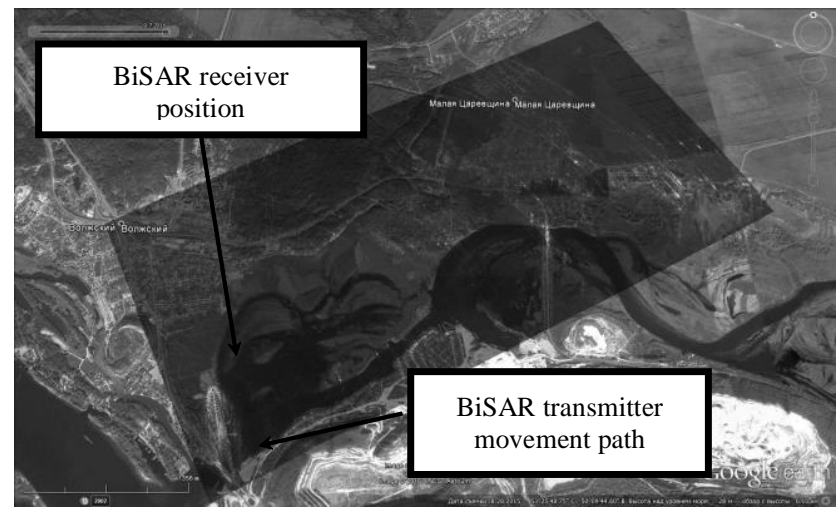

Figure 6. Scene of experiment.

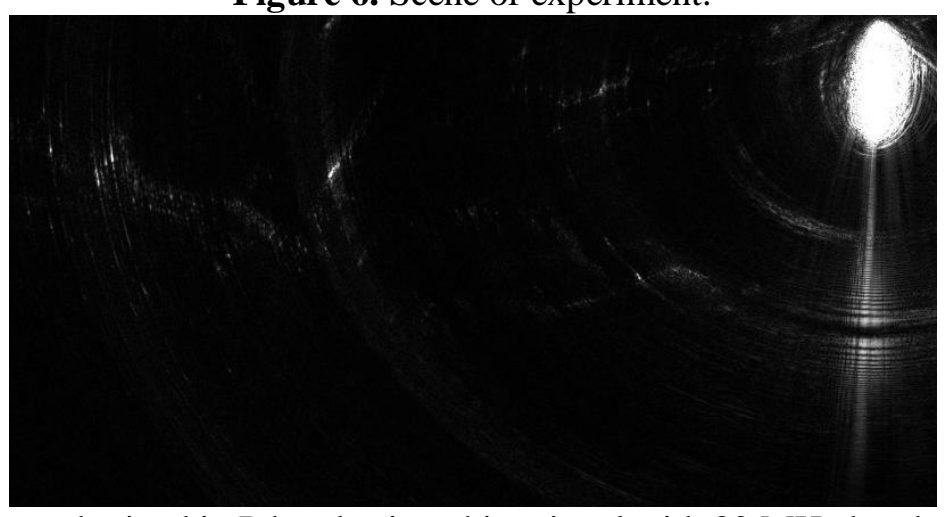

Figure 7. Radar image obtained in $\mathrm{P}$ band using chirp signal with $30 \mathrm{MHz}$ bandwidth; pixel resolution is $1.5 \times 1.5 \mathrm{~m}$; synthesis time is $40 \mathrm{sec}$.

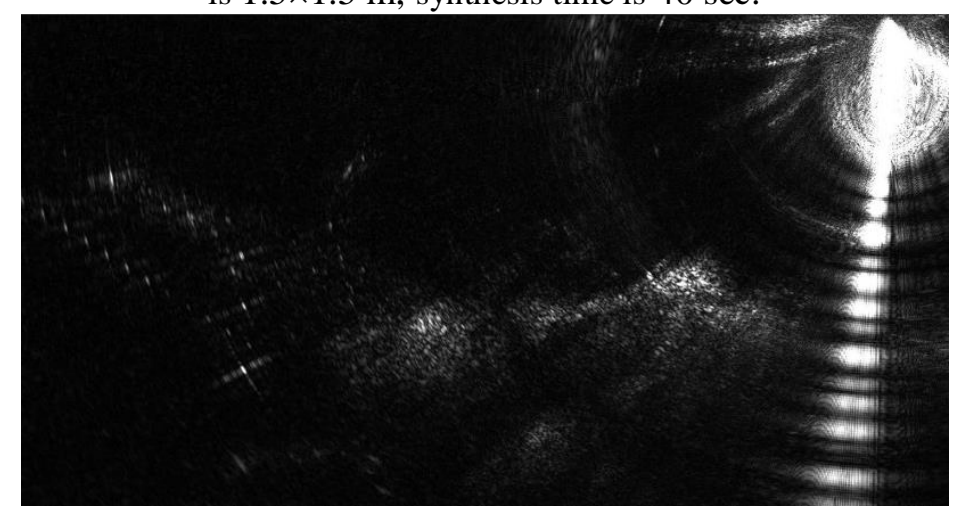

Figure 8. Radar image obtained in VHF band using PSK signal with $25 \mathrm{MHz}$ bandwidth; pixel resolution is $1.5 \times 1.5 \mathrm{~m}$; synthesis time is $40 \mathrm{sec}$.

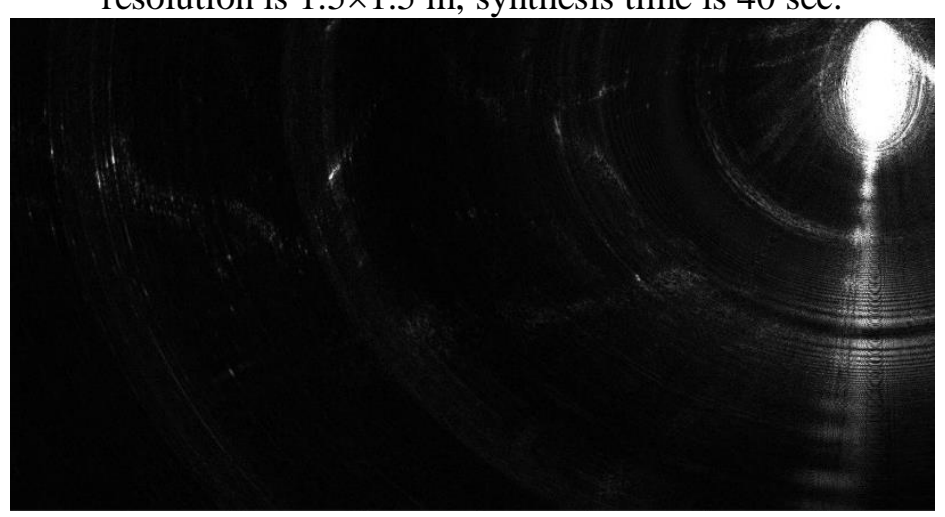

Figure 9. Radar image obtained by combining P and VHF bands; bandwidth is $80 \mathrm{MHz}$; pixel resolution is $1.5 \times 1.5 \mathrm{~m}$; synthesis time is $40 \mathrm{sec}$. 

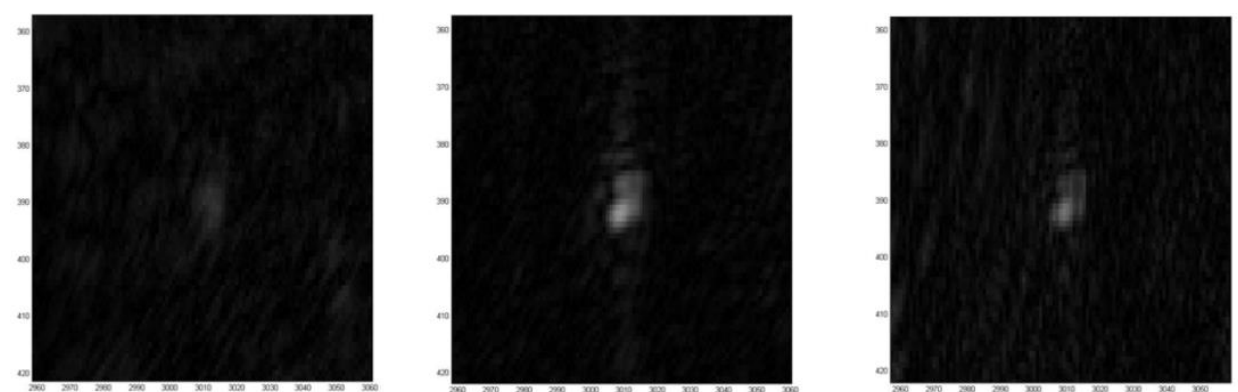

Figure 10. Radar image of the first target, left to right: VHF band, $\mathrm{P}$ band, VHF+P bands, pixel resolution is $1.5 \times 1.5 \mathrm{~m}$.
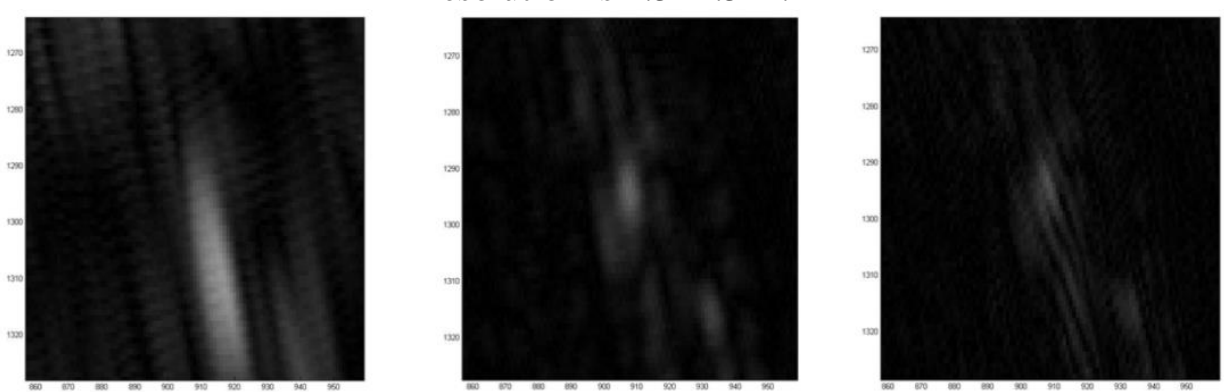

Figure 11. Radar image of the second target, left to right: VHF band, $\mathrm{P}$ band, VHF+P bands, pixel resolution is $1.5 \times 1.5 \mathrm{~m}$.

Fig. 7 and 8 show radar images in P and VHF bands respectively obtained during the ground-based experiment; pixel resolution is $1.5 \times 1.5 \mathrm{~m}$; synthesis time is $40 \mathrm{sec}$. Fig. 7 shows the image with 30 $\mathrm{MHz}$ bandwidth that corresponds to potential spatial resolution of 5 meters and Fig. 8 shows the image with $25 \mathrm{MHz}$ bandwidth that corresponds to potential spatial resolution of 6 meters (slant range resolution).

Fig. 9 shows the combined radar image for $\mathrm{P}$ and VHF bands with corresponding potential spatial resolution of 3 meters (slant range resolution). Fig. 10 and 11 show the matching fragments of the radar images in $\mathrm{VHF}, \mathrm{P}$ and $\mathrm{VHF}+\mathrm{P}$ bands that confirm the resolution increase.

During the experiment the area was observed with a small altitude (5-7 degrees), thus, radar images show only the taller objects, such as masts, power transmission lines, tall buildings, lone tall trees, wooded areas, etc.

The results of the experiment allow us to conclude that there exists a possibility to create a complex of onboard and ground-based equipment together with algorithms and software for the BiSAR system that allows to obtain radar images for VHF and $\mathrm{P}$ bands and produce high resolution images for combined $\mathrm{VHF}+\mathrm{P}$ band with spatial resolution up to 2 meters.

For the year 2018 we are planning to conduct a similar experiment using UAV (octocopter drone) to carry the mobile BiSAR transmitter.

\section{Conclusion}

The results of the full-scale ground experiment show that using both VHF and P bands with respective resolutions of 6-10 and 6 meters it is possible to obtain a combined radar image with range resolution of 3-6 meters. Thus, the proposed technology demonstrates the possibility of combining VHF and P band probing signals to increase the spatial resolution of bistatic SAR systems.

\section{References}

[1] Hellsten H and Ulander L M H 2000 Airborne Array Aperture UWB UHF Radar - Motivation and System Considerations IEEE Aerospace and Electronic Systems Magazine 15(5) 35-45

[2] Ulander L, Pierson W, Lundberg M, Follo P, Frolind P-O and Gustavsson A 2004 CARABASII SAR Change Detection Performance on Ground Targets concealed by Foliage Proceedings of 5th European conference on synthetic aperture radar 297-300 
[3] de Macedo K A C, Shiroma G H X, Wimmer C and Moreira J R 2015 Monitoring under foliage with airborne SAR Proceedings of IEEE Intl. Radar Conference At 499-505

[4] Goriachkin O V, Zhengurov B G, Bakeev V B, Baraboshin A Y, Nevski A V and Scorobogatov E G 2015 Bistatic synthetic aperture radar P band for small satellite "AIST-2" Electrosvyaz 8 34-39 (in Russia)

[5] Goriachkin O V 2016 Earth Radar Remote Sensing Center in PSUTI Vestnik svyazi 2 24-26 (in Russia)

[6] Zherdev D A, Kazanskiy N L and Fursov V A 2015 Object recognition in radar images using conjugation indices and support subspaces Computer Optics 39(2) 255-264 DOI: 10.18287/0134-2452-2015-39-2-255-264 